\title{
STUDIES OF THE SOLAR X-RAY SPECTRUM AS A FUNCTION OF POSITION ON THE DISK
}

\author{
D. H. BRABBAN, W. M. GLENCROSS and J. R. H. HERRING \\ Mullard Space Science Laboratory, University College, London, U.K.
}

Work has been in progress for many years at University College London to determine how the intensity spectrum of emitted X-rays changes as a function of time and position on the solar disk. Early spectroheliographs consisted of grazing incidence parabolic mirrors which focussed radiation onto proportional counters from which spectral data were obtained. Such instruments, built in collaboration with the University of Leicester Group, have been flown on two rockets (Negus et al., 1969) and on the OSO V satellite (Herring et al., 1971).

The spectral information obtained from proportional counters in the wavelength range studied $(8-20 \AA)$ is poor and it has not proved possible to resolve spectral lines. However it has been possible to produce daily contour maps of the X-ray flux emitted within a wavelength band several Ångstroms wide over a period of nearly two years.

To improve on this spectral resolution, rocket payloads have been produced containing Bragg crystal spectrometers. The spectral resolution of the instrument is obtained by placing a multi-grid mechanical collimator in front of each crystal. When the rocket payload is pointed at the solar disk, only radiation from a field nine arc minutes in diameter falls on the crystal. The crystal has to be scanned through the spectral range $6-25 \AA$ when the rocket is stabilized to point at a specific solar feature. Then the rocket is offset to another interesting feature and the spectral scan repeated. During a rocket flight it is only possible to observe the emission from about half-adozen solar regions so that even with the coarse spectral resolution chosen it is not possible to build a complete spectroheliogram.

To obtain a spectroheliogram in the radiation of a single spectral line with a spatial resolution of about one arc minute could prove to be extremely difficult with the Bragg Spectrometer preceded by a multi-grid collimator for two reasons:

(1) The crystal has a low reflection efficiency unless it is set accurately on the peak of the rocking curve for the particular spectral line. This means that it has to have a well aligned lattice structure over the whole surface which needs to be of area about $100 \mathrm{~cm}^{2}$. In addition, it is necessary to set the crystal accurately at the Bragg angle with respect to the axis of the collimator.

(2) A collimator of angular resolution one arc minute has to be built from many grids aligned to a fine tolerance in a poor thermal environment during flight.

A solution to the problems is to place a rotation collimator (Willmore, 1970) in front of the crystal. Wavelength resolution is obtained by moving the crystal and detector in the conventional manner, but at the same time the incident beam is modulated by a moving system of grids. From the modulated signal it is possible to reconstruct an image of the sun in the radiation emitted in each spectral line. With this 
instrument, the field of view is not restricted to a small angle in the way it would be with a conventional collimator. As a result, an image of that part of the sun which radiates the energy striking the crystal within the rocking curve can be produced after a single rapid rotation of the collimator. A flight of such a system is planned for 1971 .

\section{References}

Herring, J. R. H., Glencross, W. M., Parkinson, J. H., and Pounds, K. A.: 1971, Proc. Roy. Soc. London A321, 493.

Negus, C. R., Glencross, W. M., and Pounds, K. A.: 1969, Proc. Roy. Soc. London A317, 101.

Willmore, A. P.: 1970, Monthly Notices Roy. Astron. Soc. 147, 387. 